\title{
VLBI2010 - The TWIN radio telescope project at Wettzell, Germany
}

\section{G. Kronschnabl, T. Klügel, H. Hase}

Bundesamt für Kartographie und Geodäsie, Geodätisches Observatorium Wettzell Sackenrieder Str. 25, D-93444 Bad Kötzting, Germany

\author{
A. Neidhardt* \\ Forschungseinrichtung Satellitengeodäsie, Technische Universität München, \\ Geodätisches Observatorium Wettzell \\ Sackenrieder Str. 25, D-93444 Bad Kötzting, Germany \\ K. Pausch \\ Vertex Antennentechnik GmbH \\ Baumstr. 46-50, D-47198 Duisburg, Germany \\ W. Göldi \\ Mirad Microwave AG \\ Hofstetstr. 6, CH-9300 Wittenbach, Switzerland
}

The Twin Telescope Wettzell (TTW) Project is funded to be executed during the period of 20082011. The design of the TTW was based on the VLBI2010 vision of the corresponding IVS Working Group. In the first two project years the design passed the simulations with respect to its specifications and was approved for production. At the Geodetic Observatory Wettzell a thorough soil analysis was made in order to define the sites for the towers of the new radio telescopes. Meanwhile the construction work has begun and acceptance tests of several telescope parts, e.g. azimuth bearings, took place. The full assembly of the radio telescopes is scheduled for the next two years. In parallel to the construction work at the Wettzell site, the design work for the different feed options progressed.

The 10th European VLBI Network Symposium and EVN Users Meeting: VLBI and the new generation of radio arrays

September 20th - 24th, 2010

Manchester, $U K$

\footnotetext{
* Speaker.
} 


\section{Introduction}

On the basis of the International VLBI Service (IVS) requirements for geodetic radio telescopes the Bundesamt für Kartographie und Geodäsie (BKG) has launched the TWIN project at Wettzell (see [2]). Within this two rapidly moving radio telescopes are built, which will be equipped with a broadband receiving system including the two geodetically used frequency bands S and X. Both telescopes have been designed for continuous operations over 24 hours 7 days per week. As array both telescopes can be additively interconnected, so that the effective receiving surface corresponds approximately to that of the current $20 \mathrm{~m}$ radio telescope at the Wettzell observatory. The radial symmetric reflector concept combines the advantages of a dual offset antenna, as low noise temperature, with the advantages of a Cassegrain or Gregory antenna regarding the mechanical stability, the control possibility and the weight.

The technical data of the telescopes are:

- Diameter Main reflector: $13.2 \mathrm{~m}$

- Diameter sub-reflector: $1.48 \mathrm{~m}$

- Ring Focus Design with $\mathrm{f} / \mathrm{D}=0.29$

- Surface quality of the reflectors: $<0.2 \mathrm{~mm}$ RMS

- Way length error : $<0.3 \mathrm{~mm}$

- Surface quality of the panel: $<0.065 \mathrm{~mm}$ RMS

- ALMA mounting with angular velocities

- Angular velocities of $12 \breve{r} / \mathrm{s}$ in azimuth and $6 \check{r} / \mathrm{s}$ in elevation

- Acceleration: $\mathrm{Az} / \mathrm{El}=3 \check{r} / \mathrm{s} 2$

- Ranges of rotation: Azimuth 540ř, Elevation 0-115ř

- Balanced outrigger

- Excellent bearing

- 27Bit Encoder: 0.0003ř resolution

- Sub-reflector adjustable via a hexapod

\section{The ring focus design}

The specially designed reflector uses a radial symmetric design. The advantage of this ring focus antenna (see fig. 1) is a reflection of the rays from the outer main reflector regions into the center of the sub-reflector giving a better illumination of the feed horn. This design is properly suited for broadband feed horns, which need a wider opening angle. Therefore the feed must be positioned close to the sub-reflector, which is specially designed for such a setup.

\section{Structure optimization}

The TWIN radio telescopes were especially developed for geodetic applications. Therefore it is essential to achieve extremely low deviations in path length between main reflector, phase center of the feed horn and axis intersection of the telescope (a path length error of $0.3 \mathrm{~mm}$ was specified!). Elaborate simulations using the Finite Element Method were performed to achieve a 


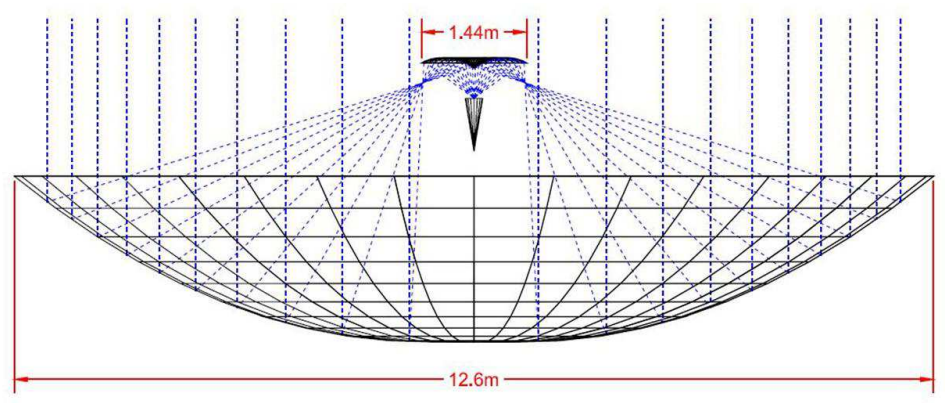

Figure 1: The ring focus design

very torsion-resistant and solid supporting structure. All components from the main reflector to the concrete tower were optimized for occurring load cases (wind, operating weight, temperature) to guarantee the required stability. Additionally the sub-reflector is positioned with a hexapod for gravity corrections.

\section{Microwave receiving systems}
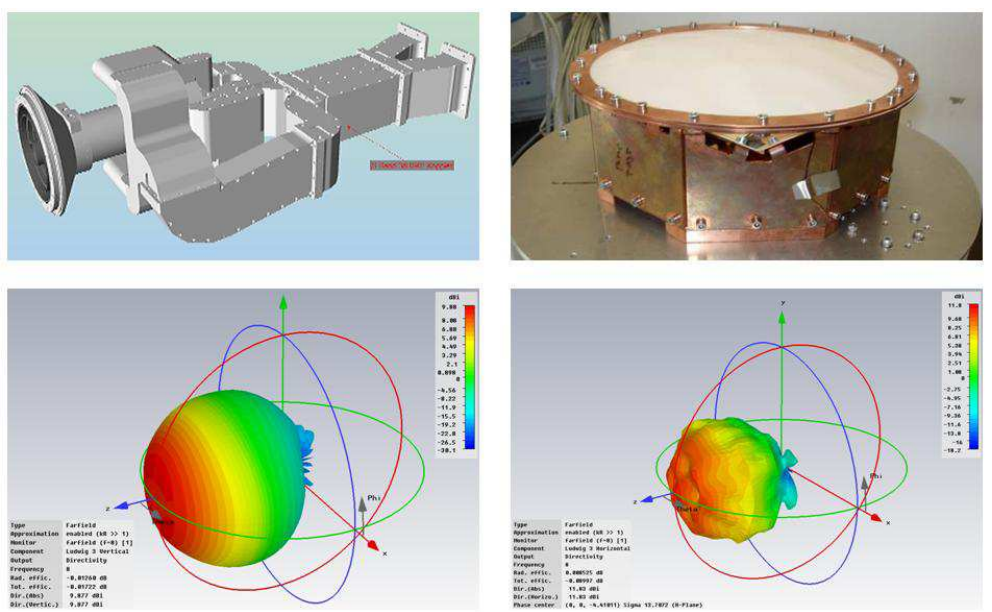

Figure 2: The proposed feed horns: a tri-band coaxial feed and the Eleven feed

The VLBI2010 concept designed a broadband receiving bandwidth of 2 - $14 \mathrm{GHz}$, with the option to integrate the Ka-band $(28-36 \mathrm{GHz})$. To realize these ideas new receiving systems have to be developed, which will enable homogeneous illumination of the main reflector. They must have a stable phase center and a system-noise temperature below $50 \mathrm{~K}$.

BKG has commissioned a tri-band feed horn (see 2 on the left side) that is able to work in the two geodetic frequency bands (S/X-Band), and also in the Ka band (see [1]). It will be possible to participate in all standard VLBI and deep space network observations and to make a substantial contribution to the improvement of the celestial reference frame in the Ka band.

The Elevenfeed (see 2 on the right side) of Prof. Kildal (Chalmers University, Sweden) presently offers the best preconditions for the reception of a continuous frequency range of 2 to 
$14 \mathrm{GHz}$ (see [3]). Extensive simulations showed good performance of the feed up to ca. $10 \mathrm{GHz}$. From $10-14 \mathrm{GHz}$ the performance is also suitable. The ohmic attenuation of the copper lines and the differential outputs pose some problems. To solve them cryogenic cooling as well as several special low-noise-amplifiers are necessary.

The microwave converter is designed for four $1 \mathrm{GHz}$ broad frequency bands, which then can be recorded with digital data recording systems. Because of the broadband receiving, which covers approximately three octaves, two frequency converters are required.

\section{Locations of the new telescopes}

For the location decision for the two TWIN telescopes extensive soil specimens and geological surveys were done. The goal was to find two locations for the two telescopes which offer as good geological stability as the $20 \mathrm{~m}$ radio telescope. This has an extraordinarily low azimuth axis deviation. In total more than 12 drillings were made to determine the geological conditions. Special attention was turned to set the height of the elevation axis onto the same level for an additional system monitoring. A suitable location was found close to the observatory, which simplifies the connection of the new telescopes according to the local survey, the infrastructure and the operational tasks.

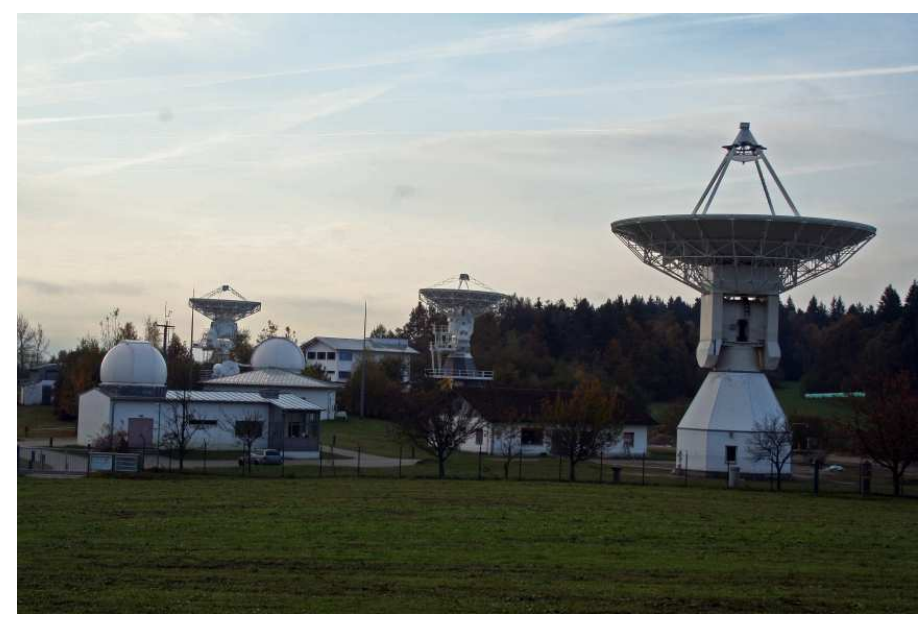

Figure 3: The new sky line of the Geodetic Observatory Wettzell: in the background are the new TWIN telescopes

\section{Control and operations}

Both telescopes have been designed for continuous operations over 24 hours 7 days per week. Therefore special attention was turned to the control concept and the servo system. All components were designed for high availability, precise tracking and 1000 source observations per day. Following this strategy continuous geodetic time series can be generated with higher accuracy. Especially the increased number of sources and an efficient observation improves the tropospheric corrections. According to this new observation strategies must be developed where the telescopes can operate 
parallel on different sources, additiv on the same sources or in single telescope mode, to maintain the non-operative one.

Other new techniques will allow to increase the e-VLBI sessions and to run the observations with remote control or completely unattended. The new operational building is designed to allow such new control modes. It offers possibilities for local fringe test and zero baseline correlators as well as the connectivity and control room to run different sites from there. This is very important for the operations of the telescopes at the Transpotable Integrated Geodetic Observatory (TIGO) in Concepción, Chile and at the German Antarctic Receiving Station (GARS) O'Higgins, which are operated by personnel stuff of Wettzell. To realize such remote control scenarios the e-control software will be used, which is developed by a team of the observatory[4].

\section{Summary and outlook}

Overall the described setup is the first complete and rigorous realization of the visions of VLBI2010 (see [5]). The telescopes are in the final construction phase. All of the high frequency components are commissioned and are under construction now. The digital broad band acquisition system is under test. The new antennas will be tested for operations in the year 2011. Colocated with the existing $20 \mathrm{~m}$ radio telescope and with the other instruments for space geodesy the new project is a pathfinder technology for the new proposed stations within the Global Geodetic Observing System (GGOS).

\section{References}

[1] W. Göldi, Evaluation of Suitable Feed Systems, IVS VLBI 2010 Workshop on Future Radio Frequencies and Feeds,

http://www.fs.wettzell.de/veranstaltungen/vlbi/frff2009/Part3/ 10-Goeldi_Workshop21.pdf (2009).

[2] H. Hase, R. Dassing, T. Klügel, G. Konschnabl, A. Neidhardt, P. Lauber, R. Kilger, K. Pausch, Twin Telescope Wettzell (TTW) - A VLBI2010 project, IVS VLBI 2010 Workshop on Future Radio Frequencies and Feeds,

http://www.fs.wettzell.de/veranstaltungen/vlbi/frff2009/Part8/ ttwvlbi2010e.pdf (2009).

[3] P.-S. Kildal, Characterization of Feeds for Radio Telescopes, IVS VLBI 2010 Workshop on Future Radio Frequencies and Feeds,

http://www.fs.wettzell.de/veranstaltungen/vlbi/frff2009/Part3/ 00-Tutorial-Wettzel-Kildal-090317cr.pdf (2009).

[4] A. Neidhardt, M. Ettl, C. Plötz, M. Mühlbauer, R. Dassing, H. Hase, S. Sobarzo, C. Herrera, W. Alef, H. Rottmann, e-control: new concepts for remote control of VLBI-telescopes and first experiences at Wettzell, The 8th International e-VLBI Workshop, EXPReS09, PoS (EXPReS 09 ) 038 (2009).

[5] Niell, Arthur, et. al., IVS Memorandum 2006-008v01. "VLBI2010: Current and Future Requirements for Geodetic VLBI Systems", http://ivscc.gsfc.nasa.gov/publications/memos/index.html (Sept. 2004). 